Cronfa - Swansea University Open Access Repository

This is an author produced version of a paper published in :

International Journal of Fatigue

Cronfa URL for this paper:

http://cronfa.swan.ac.uk/Record/cronfa5830

http://dx.doi.org/10.1016/j.jifatigue.2009.02.014

This article is brought to you by Swansea University. Any person downloading material is agreeing to abide by the terms of the repository licence. Authors are personally responsible for adhering to publisher restrictions or conditions. When uploading content they are required to comply with their publisher agreement and the SHERPA RoMEO database to judge whether or not it is copyright safe to add this version of the paper to this repository. http://www.swansea.ac.uk/iss/researchsupport/cronfa-support/ 


\title{
The effect of microstructure and texture on mechanical properties of Ti6-4
}

\author{
MT Whittaker ${ }^{[1]}$, WJ Evans ${ }^{[1]}$, R Lancaster ${ }^{[1]}$, W Harrison $^{[1]}$, PS Webster ${ }^{[2]}$
}

[1] Swansea University, Singleton Park, Swansea. SA2 8PP

[2] Rolls-Royce plc, Elton Road, Derby DE24 8BJ

\begin{abstract}
The widely used titanium alloy, Ti6-4, is available in several different microstructural conditions, with variations being due to a number of factors including processing route and subsequent heat treatments. These differing microstructures can produce significant variations in the fatigue life of the material and as such it is essential that predictions can be made about the fatigue performance based on the microstructural condition. The paper examines six microstructural variations of Ti6-4 and seeks to identify trends within strain control data, and the subsequent effect on notched specimen behaviour, used to characterise the effect of stress raising features in engineering applications.
\end{abstract}

\section{Introduction}

Since the 1950s the aerospace sector has benefited from a long standing and fruitful relationship with titanium alloys. The relatively low density and high strength to weight ratio of these alloys provides designers with the ability to produce lightweight durable materials for low to mid temperature applications, such as those required in the forward stages of the gas turbine engine.

Ti6-4 was one of the early titanium alloys to find widespread applications in the aerospace sector and is an alpha-beta alloy which is commonly supplied in a number of different microstructural conditions. A wide variety of studies are available in the literature which focus on the differences in high cycle fatigue life or crack propagation rate that such microstructures can produce. However, less is known about the effect of microstructural variations on the low cycle fatigue life of the alloy, particularly under strain control conditions typical of many engine applications. 
The matter is often further complicated by the presence of crystallographic textures produced during thermo-mechanical processing. It has been shown that texture effects can have a marked effect on mechanical properties ${ }^{[1,2]}$ and Lutjering ${ }^{[3]}$ has shown that there is only limited scope for separating microstructural and texture evolution during material processing and subsequent heat treatments. The combination of microstructure and texture often results in large variations in fatigue life, which under strain control conditions may provide designers with difficult life prediction issues. The present paper seeks to evaluate the strain-life LCF performance of a number of microstructural variants of Ti6-4 and relate the trends seen to such issues as primary alpha grain size, primary alpha volume fraction, morphology of transformed product and crystallographic texture. An alternative publication ${ }^{[4]}$ addresses the influence of texture on these loading conditions, and the prediction of notch fatigue life based purely on strain control data. The current paper seeks to make use of this work in separating the effects of texture and microstructure. Furthermore the paper utilises established predictive techniques such as Walker strain ${ }^{[5]}$ and Coffin-Manson ${ }^{[6,7]}$ to assess the effects of stress concentrations on the microstructural variants. A predictive model based on these concepts is presented.

\section{Material}

Ti6Al-4V is one of the most extensively used titanium alloys due to its favourable balance of mechanical properties, including moderately high tensile strength, good fatigue properties with intermediate fracture toughness ${ }^{[8]}$. The range of microstructural conditions is determined by the thermo-mechanical processing history of the material. This study utilises six variants of the alloy, Figure 1, and these alloys are described in Table 1:-

Clearly noticeable from Figure 1 are the distinctly different microstructures and textures of the tested variants. Variants A, B, C and F show essentially equiaxed primary alpha grains that constitute large percentages of the microstructure. Variant D is a more typical bimodal microstructure with primary alpha grains isolated within transformed product. Variant $\mathrm{E}$ is typical of a material processed above the beta transus, with isolated poles of texture and a Widmanstatten microstructure.

Variants $\mathrm{B}$ and $\mathrm{E}$ show the strongest levels of texture, although previous work has shown that this will not always results in the greatest anisotropy in mechanical properties $^{[9]}$. 


\section{Experimental procedure}

The $6 \mathrm{~mm}$ diameter specimens were tested under strain control to BS7270 ${ }^{[10]}$ on a Mayes servo hydraulic machine using a controlling MTS high temperature extensometer with a gauge length of $10 \mathrm{~mm}$. The experiments were completed using a (1-1-1-1) trapezoidal waveform at $15 \mathrm{cpm}$ with $\mathrm{R}$ ratios of -1 , and 0 . The $\mathrm{R}=-1$ tests provided open hysteresis loops for use in the modelling work. Stress-strain loops were recorded by an in-house data logging system which also allowed the recording of peak and minimum stresses so that a graph of stress relaxation as a function of cycles could be recorded.

Fatigue tests on double edged notch (DEN) specimens, Figure 2, were undertaken on a Mayes servo hydraulic test machine, with the development of fatigue cracks monitored by a standard potential drop technique ${ }^{[11]}$. Testing of the titanium alloy Ti6246 using DEN specimens has allowed a calibration to be made between voltage change and crack length, Figure 3. Although the calibration reveals a 'coarse' indication of initiation, tracing the voltage graph backwards reveals that actual crack growth begins at $40-50 \%$ of the total life. Earlier studies in Ti6-4 have revealed similar trends.

It should be noted that variants D and E were tested at Swansea University many years prior to the other variants and as such data logging facilities were not as comprehensive as the current data acquisition software. As such most of the results from these alloys are presented only in terms of stress-life and strain-life since hysteresis loop data is not available.

\section{Results}

Clearly in this work it is important to compare like materials as much as possible. Whilst it is acknowledged that there may be significant performance variations in materials such as Ti6-4 ${ }^{[12]}$ due to alloy chemistry and its control, it is assumed in the current work that chemistry effects are negligible and variations are due to the effects of microstructure or crystallographic texture. 


\section{Texture effects}

Previous work ${ }^{[9]}$ has shown how texture affects the strain control behaviour of titanium alloys. The availability of prismatic slip at room temperature has a major influence on the cyclic softening behaviour of the material, which consequently affects stabilised mean stress, stabilised stress range and subsequent life. Slip on prismatic planes is favoured at room temperature in these alloys since it has a significantly lower critically resolved shear stress (c.r.s.s.) than basal slip. The previous work has also shown that in a typical uni-directionally rolled titanium plate, relatively light textures of $x 3$ random can lead to significant variations in strain-life. It is also acknowledged, however, that crack development in these alloys generally occurs on basal planes, and as such basal plane orientation is important.

In this work material orientations have been chosen so that differences in texture are kept to a minimum when comparing across microstructural variants. However, clearly there are variations, and a detailed knowledge of texture effects on strain control behaviour in this material is required to separate texture and microstructural influences. A more in-depth analysis of texture effects can be found in an alternative publication $^{[4]}$.

Figure 4 illustrates the effects that a relatively weak texture can have on strain-life, with upper and lower bounds for each variant indicated. Variants A and B are both unidirectionally rolled plates, and hence show relatively strong textures. When viewed on a strain range basis it can be seen in both variants that Rolling Direction (RD) specimens show a significantly longer life than Transverse Direction (TD) specimens. However, when analysing the data on a stabilised stress basis, Figure 5, orientation effects in the two sets of data are negligible. This indicates the importance of slip and available slip planes in the early part of the test, since they will influence stress redistribution and the eventual stabilised stress that clearly controls the lives of the specimens. Figure 6 illustrates this point clearly for variant A, in considering a strain control test with a peak strain of $1.4 \%$. RD specimens have more prismatic slip planes available than the TD specimens, and consequently the stress relaxes more in the early part of the test. The fact that the TD specimens have a higher modulus due to a greater number of basal planes perpendicular to loading direction (See pole figure in Figure 1) results in a higher stress range for the test. As a consequence of this increased stress 
range, Figure 5 indicates that the TD specimen will now show a reduced life in comparison to a RD specimen cycled to the same peak strain.

The fact that variant A shows longer fatigue lives than variant B in either orientation is a result of the smaller effective structural unit size of variant $\mathrm{A}$, as discussed in the following section. The result however indicates that the stress life of the material is controlled by microstructure, whereas texture produces a secondary effect, which is realised only as a variation in strain life with orientation, as a result of stress relaxation.

\section{Microstructural variations}

Figure 7 compares the six variants on a stabilised stress-life basis, with lines superimposed to indicate data trends. It can be seen that the materials show considerably different fatigue lives. As stated previously, since the stress-life behaviour of the material is now being considered, the variation must be a result of microstructure, rather than texture, which only significantly influences strain-life. In order to compare the materials with as few variables as possible, the plate materials $(\mathrm{A} \& \mathrm{~B})$ are now considered in the RD orientation, the bar stock (variant $\mathrm{F}$ ) along the length of the bar, and the disc materials in the radial direction (variants $\mathrm{C}, \mathrm{D} \& \mathrm{E}$ ). These orientations are the most similar in terms of texture.

Clearly in the current investigation, the microstructural variants have changed the inherent strength of the material, with trends clearly visible, even when scatter in the data is considered. This can be attributed largely to grain size effects through the HallPetch relationship, although the correlation is not particularly strong. It is interesting to take a variation of the Hall-Petch relationship, through the stabilised stress range at

$10^{5}$ cycles from Figure 7, and plotting against the square root of the grain size. While there is a general trend for larger grained variants to show a poorer fatigue performance, the relationship is not strong, with an $\mathrm{R}$ squared value of only 0.557 . What is required is a more detailed analysis of the material, considering the effective structural unit (ESU) size of each variant. Sackett et al ${ }^{[13]}$ have described an ESU as an area of common crystallographic orientation which need not be related to the primary alpha grain size of the material. Whilst there is still debate over the nature and definition of these units, a basic application here is beneficial. 
If we consider the Ti6-4 variants described in the current work on a stress-life basis, it becomes clear that the seemingly disparate behaviour can be described by this approach. The poorest performance is shown by variant E, with the Widmanstatten microstructure. Resistance to fatigue crack initiation depends on the weakest link. Within this microstructure the platelets are orientated at a variety of angles, some of which will be suitably orientated for slip. This type of structure will typically have large areas of common orientation, which will provide long unrestricted slip paths, clearly detrimental to fatigue performance.

Variant B shows a very similar fatigue performance. The microstructure is now of relatively equiaxed large alpha grains with larger areas of common orientation available due to the strong, well defined texture. For the same reasons as variant E, this results in a relatively poor fatigue performance. Variant $\mathrm{C}$ shows a further improvement because of the finer grain size (approximately $15 \mu \mathrm{m}$ ) of the primary alpha and the fact that platelets within the transformed product are also approximately $15 \mu \mathrm{m}$. EBSD analysis has shown areas of common orientation to be limited essentially to a single primary $\alpha$ or transformed $\alpha$ grain and hence the ESU of this variant is also approximately $15 \mu \mathrm{m}$. A similar primary alpha grain size applies to variant $\mathrm{F}$, which has very low levels of transformed product. For this reason variants $\mathrm{C}$ and $\mathrm{F}$ behave similarly, due to an ESU of approximately $15 \mu \mathrm{m}$.

By attempting purely to order the materials in terms of primary alpha grain size, it is difficult to see why variants A \& D show considerably longer fatigue lives than variants $\mathrm{C} \& \mathrm{~F}$, since the grain sizes are similar. However, in both cases it is noticeable that there is considerable transformed product. Further inspection of these areas shows that the transformed product is discontinuous over length scales greater than approximately $5 \mu \mathrm{m}$, and in each case the crystallography of this product is essentially random due to more extensive cold working of the alloy. If it is considered that the fatigue performance of the material is a combination of the ESU of this product and the primary alpha grain size, it becomes clear that these variants would show the greatest fatigue performance. This is consistent with previous work performed by Stubbington ${ }^{[14]}$ which stated that for high fatigue strength the lengths of alpha/beta interfaces within the transformed product should be kept to a minimum. This can be achieved by extensive low temperature working of the alloy.

It is clear that by applying the idea of an effective structural unit, the microstructures are now ordered in terms of stabilised stress-life. At one end of the scale lie variants 
$\mathrm{B} \& \mathrm{E}$, which have large areas of common orientation allowing for extended slip that promotes fatigue crack initiation and hence, poor fatigue performance. At the other end are the structures (A\&D) involving more cold work, which show discontinuous transformed product over relatively short scales, and hence a smaller ESU, producing improved performance.

\section{Predicting notched fatigue behaviour}

Previous work ${ }^{[15]}$ has demonstrated the applicability of critical strain methods for the prediction of notched fatigue behaviour in titanium alloys. In a similar way, it has been found that for the relatively shallow notch geometries tested during this programme, the Walker strain technique ${ }^{[5]}$ is a particularly effective method for $\mathrm{R}=0$ loading conditions. Its accuracy however is based on adequate description of the stress and strain conditions at the notch root. Although the Neuber rule $(\sigma \varepsilon=$ constant) for establishing stress-strain data at the notch root is a useful approximation and has been used widely, it becomes clear that a more robust method is required.

The modelling process in this work is based solely upon strain control data. Under strain control conditions local stress redistribution processes occur during the early part of the test before peak tensile and compressive stresses stabilise for the remainder of the test. This behaviour was modelled through use of the Mroz multilayer kinematic hardening method ${ }^{[16]}$, in the form of a user subroutine within the commercial finite element package ABAQUS. Simulations of the strain control specimens were run using this subroutine and Figure 8 shows the success with which the hysteresis loops were reproduced. Modelling of the DEN specimen was achieved through a 3D 1/8 symmetrical FE model as in previous publications. ${ }^{[17]}$.

Strain control data is appropriate for predicting the behaviour of stress concentrations because of the fact that the local plastic strain is contained within a small region near the notch root where fatigue crack initiation usually occurs. It is argued that, due to constraint imposed by elastic material surrounding the plastic zone, material within the plastic zone experiences an essentially strain control type of deformation and hence predictions based on strain control data are possible.

Figure 9 shows the predictions made by the model using strain control data from variant $\mathrm{C}$ for $\mathrm{R}=0$ notch tests at $20^{\circ} \mathrm{C}$ in the same microstructural variant. The graph highlights predictions of node failures at the centre of the notch, the edge and a 
midpoint between them, as indicated in Figure 10. It can be seen that the predictions for node failure at the centre of the notch closely replicate the experimental data for freely initiated cracks. These types of predictions will tend to underestimate the measured lives of notched specimens, since the crack propagation phase will make a bigger contribution compared with a strain control specimen. This is due to the fact that once a crack forms in a strain control specimen, subsequent propagation will be extremely fast due to the increasing stress field into which it is grown. In a notched specimen, because of the stress gradient away from the notch, there is a more significant propagation phase. When a crack forms, it is growing through a decreasing stress field, and hence a significant propagation phase should be included in the total life. In fact, lives predicted by the model average $60 \%$ of the measured fatigue lives of the notched specimens. This type of ratio between initiation and propagation phases in a titanium alloy is not uncommon and has been observed in similar studies where crack initiation is measured by potential drop techniques ${ }^{[15]}$.

The model does not currently predict the effects of different microstructures. However, these effects can be explored through the Neuber rule. The difficulties of predicting notched specimen behaviour for different microstructural variations is illustrated by Figure 11, which shows predictions made using strain control data for variants A \& C for DEN specimens of each microstructural variant. It can be seen that the predictions, indicated by the lines on the graph, would both be regarded as reasonably accurate for predicting that particular variant. However, the tested specimens actually seem to behave as a single set of continuous data, a fact that is not mirrored by the predictions, particularly in the LCF regime.

This is further emphasised by Figure 12 which shows a graph of the behaviour of DEN specimens of three of the microstructural variants. The graph also includes some Round Cylindrical Notch (RCN) specimens tested in variant A. The RCN specimen compares well with the DEN since the shape of the notch in 2D is the same. It is clear that there is little difference between the microstructural variants, which is interesting since it was anticipated that differences in fatigue behaviour seen under strain control would also be revealed by the notched specimens. 


\section{Discussion}

The fact that an order of magnitude difference in strain control fatigue performance can be achieved through varying microstructure reveals the importance of understanding these effects. Previous authors ${ }^{[3]}$ have shown the difficulties associated with separating microstructure and texture effects and it has been important to isolate the effects of each in this work. Microstructural effects have been isolated through the use of materials with similar or weak textures, whereas texture effects have been isolated in variants $\mathrm{A}$ and $\mathrm{B}$, each with a relatively strong texture, by testing different sample orientations.

Clearly there is a large variation in the fatigue performance of the microstructural variants when viewed on a strain range or stabilised stress range basis. It has been shown that the differing microstructures can be analysed through an effective structural unit (ESU) approach, which orders each microstructure from poorest fatigue performance for large ESUs to the best for small ESUs. It is however clearly shown that to fully understand the performance of any of the materials, both microstructure and texture must be considered.

Microstructure fundamentally governs the fatigue performance of the material, as can be clearly seen by in Figure 7, showing fatigue performance on a stress-life basis. Crystallographic texture acts as a secondary effect, which is observed only when results are viewed on a strain-life basis. This is a consequence of the availability of prismatic planes that allow stress relaxation in the early part of the test, and control the stabilised stress range in a strain control test. This accounts for strain-life variation in the two orientations of a single microstructure

The long term aim of the work is to develop a modelling capacity that is capable of predicting the behaviour of stress concentration factors, based purely on stress-strain data. At the current stage of model development the ability to accurately model the stress-strain behaviour of the material has been demonstrated, based on the $\mathrm{Mroz}$ multilayer kinematic hardening method. Stress-strain loops are accurately replicated, and this produces confidence in the fact that the model accurately describes stress and strain conditions at the notch root. The application of numerical techniques such as the Walker strain method then allow predictions to be made for the initiation life of a notched specimen, and for the current material (variant $\mathrm{C}$ ) the predictions appear to be extremely accurate, allowing for a 60:40 ratio of crack initiation to propagation. As 
stated previously, experimental data has shown this to be a realistic prediction for this material.

Difficulties seem to occur however, when predictive techniques are applied to the different microstructural variants. Based on the strain control data, large variations in the notched fatigue performance would be expected. Figures $11 \& 12$ show that this is not necessarily the case and that the different variants show similar performance. The reason for this is most likely related to the propagation phase within the notched specimens, which is significantly longer than in the strain controlled specimens, due to the decreasing stress field of the notched specimen. Figure 13 shows a graph of crack propagation rates for three of the variants. It can be seen that propagation rates vary quite significantly between the different microstructural variants, with the larger grained variant B showing a reduced propagation rate, as would be expected due to greater deflection of the crack path. The fact that propagation rates are up to three times faster in variants $\mathrm{A} \& \mathrm{C}$ means that the benefits of an extended initiation life provided by the smaller ESUs are eradicated by the propagation phase, and each of the variants shows a similar overall fatigue performance. It should be noted however, that this is an artefact of the specimen geometry (DEN) tested, and that other geometries with different initiation/propagation life ratios will not necessarily show the same effect.

\section{Conclusions}

- The stabilised stress-life of Ti6-4 is controlled by microstructure. Materials with a large Effective Structural Unit (ESU) will show a poorer fatigue performance than materials with a small ESU.

- Crystallographic texture is of importance as a secondary effect when considering the strain-life of the material. Prismatic slip in the early part of the test allows for varying degrees of stress relaxation, which in turn influences fatigue performance.

- Transformed product has a significant influence on the behaviour of the material, with fine, discontinuous grains aiding fatigue performance.

- It is possible to make accurate predictions about the initiation life of notched specimens through the use of predictive techniques, such as the Walker strain 
method. However, it should be acknowledged that a significant propagation phase can exist, and this may be influential in the behaviour of these specimens

- A modelling capability has been established which has successfully replicated material behaviour in variant $\mathrm{C}$ of this alloy, and provided accurate predictions of the fatigue life of notched specimens. Further development of the model is required to explore the modelling of microstructural variants and crack propagation.

\section{Acknowledgments}

The authors work like to thanks Rolls-Royce plc for financial support and technical expertise for the duration of this programme.

\section{References}

[1] M. Peters, A. Gysler, G. Lutjering, "Influence of texture on fatigue properties of Ti6-4”, Metallurgical Transactions A Vol 15A Aug 1984 pp 1597-1605

[2] M.R. Bache, W.J. Evans, V. Randle, R.J. Wilson, "Characterisation of mechanical anisotropy in Titanium alloys" Material Science \& Engineering A257 (1998) pp 139-144

[3] G. Lutjering, "Influence of processing on microstructures and mechanical properties of $\alpha+\beta$ Titanium alloys", Material Science \& Engineering A243 (1998) pp $32-45$

[4] M T Whittaker, W J Evans, "The influence of texture on notched specimen behaviour in a titanium alloy", Submitted to International Journal of Fatigue 2009

[5] K Walker, "The effect of stress ratio during crack propagation and fatigue for 2024-T3 and 7075-T6 aluminum" Effects of Environment and Complex Loading History on Fatigue Life, ASTM STP 462, 1970, pp1-14,

[6] LF Coffin, "Fatigue at High Temperatures", ASTM STP520, 1973, pp744782 ,

[7] SS Manson, "Fatigue: A complex subject-some simple approximations", J. Experimental Mechanics, Vol. 5, No 7., 1965, pp 193-226,

[8] TIMET Website, www.timet.com 
[9] WJ Evans, JP Jones, MT Whittaker, “Texture effects under tension and torsion loading conditions in titanium alloys", International Journal of Fatigue, Vol 27 (1012) 2005 , pp 1244-1250,

[10] BS7270, "British standard method for constant amplitude strain controlled fatigue testing", British Standards Institution, 1990

[11] M.A. Hicks, A.C. Pickard, "A comparison of theoretical and experimental methods of calibrating the electrical potential drop technique for crack length determination", International Journal of Fracture 20 (1982) pp 91-101

[12] W.J. Evans, M McElhone, D. Rugg, "Designing for variability in fatigue performance", $11^{\text {th }}$ International conference on titanium, Kyoto 2007, pp1337-1340

[13] E.E. Sackett, L. Germain, M.R. Bache, "Crystal plasticity, fatigue crack initiation and fatigue performance of advanced titanium alloys", International Journal of Fatigue, Volume 29, Issues 9-11, September-November 2007, Pages 2015-2021

[14] C.A. Stubbington, "Metallurgical aspects of fatigue \& fracture in Titanium alloys", AGARD conference No 185 (1976) pp 140-158

[15] M.T. Whittaker, W.J. Evans, P.J. Hurley and D. Flynn "Prediction of notched specimen behaviour at ambient and high temperatures in Ti6246", International Journal of Fatigue, Volume 29, Issues 9-11, September-2007

[16] Z. Mroz, "An attempt to describe the behavior of metals under cyclic loads using a more general work hardening model” Acta Mech. 7, (1969), pp 199-212.

[17] P.J. Hurley, M.T. Whittaker, P. Webster, W.J. Evans, “A methodology for predicting creep/fatigue crack growth rates in Ti 6246", International Journal of Fatigue, Volume 29, Issues 9-11, September-November 2007, Pages 1702-1710 
Table 1: Description of microstructural variants shown in Figure 1.

\begin{tabular}{|c|c|c|c|c|c|}
\hline & $\begin{array}{l}\text { Manufacturing } \\
\text { route }\end{array}$ & $\begin{array}{l}\text { Primary } \\
\text { alpha } \\
\text { volume } \\
\text { fraction } \alpha_{\mathrm{p}}\end{array}$ & $\begin{array}{l}\text { Primary alpha } \\
\text { grain size }\end{array}$ & Texture type & $\begin{array}{l}\text { Maximum } \\
\text { intensity } \\
\text { (x random) }\end{array}$ \\
\hline Variant A & UD rolled & $80 \%$ & $20 \mu \mathrm{m}$ & Basal/Transverse & $\mathrm{x} 3$ \\
\hline Variant B & UD rolled & $80 \%$ & $30 \mu \mathrm{m}$ & Transverse & $\mathrm{x} 15$ \\
\hline Variant C & Forged disc & $60 \%$ & $10 \mu \mathrm{m}$ & Random & $\mathrm{x} 2$ \\
\hline Variant D & Forged disc & $20 \%$ & $10-15 \mu \mathrm{m}$ & Random & $\mathrm{x} 2$ \\
\hline Variant E & Forged disc & $0 \%$ & $\begin{array}{l}\text { Fully } \\
\text { transformed }\end{array}$ & Random & $\mathrm{x} 10$ \\
\hline Variant F & Bar stock & $90 \%$ & $15 \mu \mathrm{m}$ & Basal & $\mathrm{x} 5$ \\
\hline
\end{tabular}




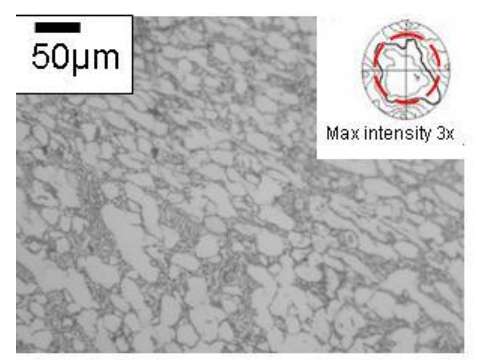

Variant A

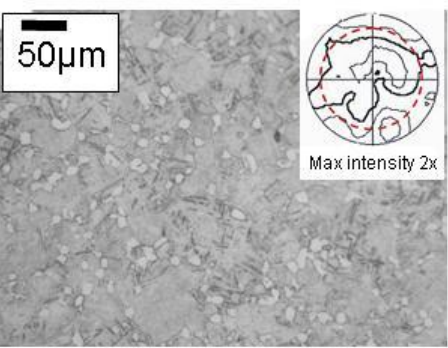

Variant D

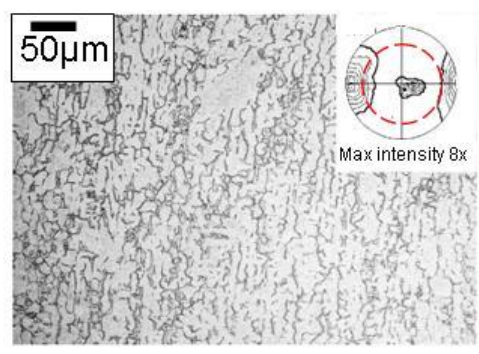

Variant $B$

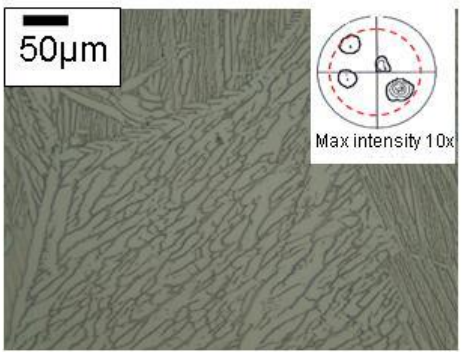

Variant $E$

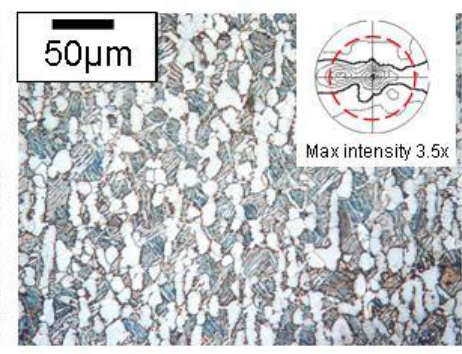

Variant C

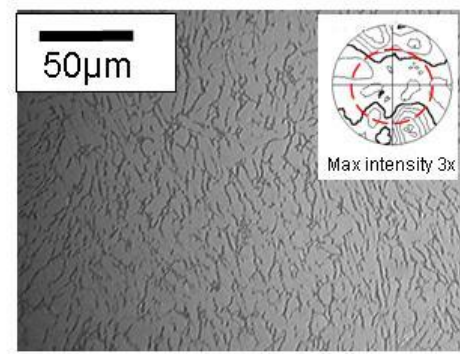

Variant F

Figure 1: Micrographs and pole figures of six microstructural variants of Ti6-4 (a) Uni-directionally rolled non-heat treated plate (b) Uni-directionally rolled heat treated plate (c) Disc forging proprietary heat treatment (d) Disc forging, alpha-beta heat treatment (e) Disc forging, beta heat treatment (f) Bar stock 


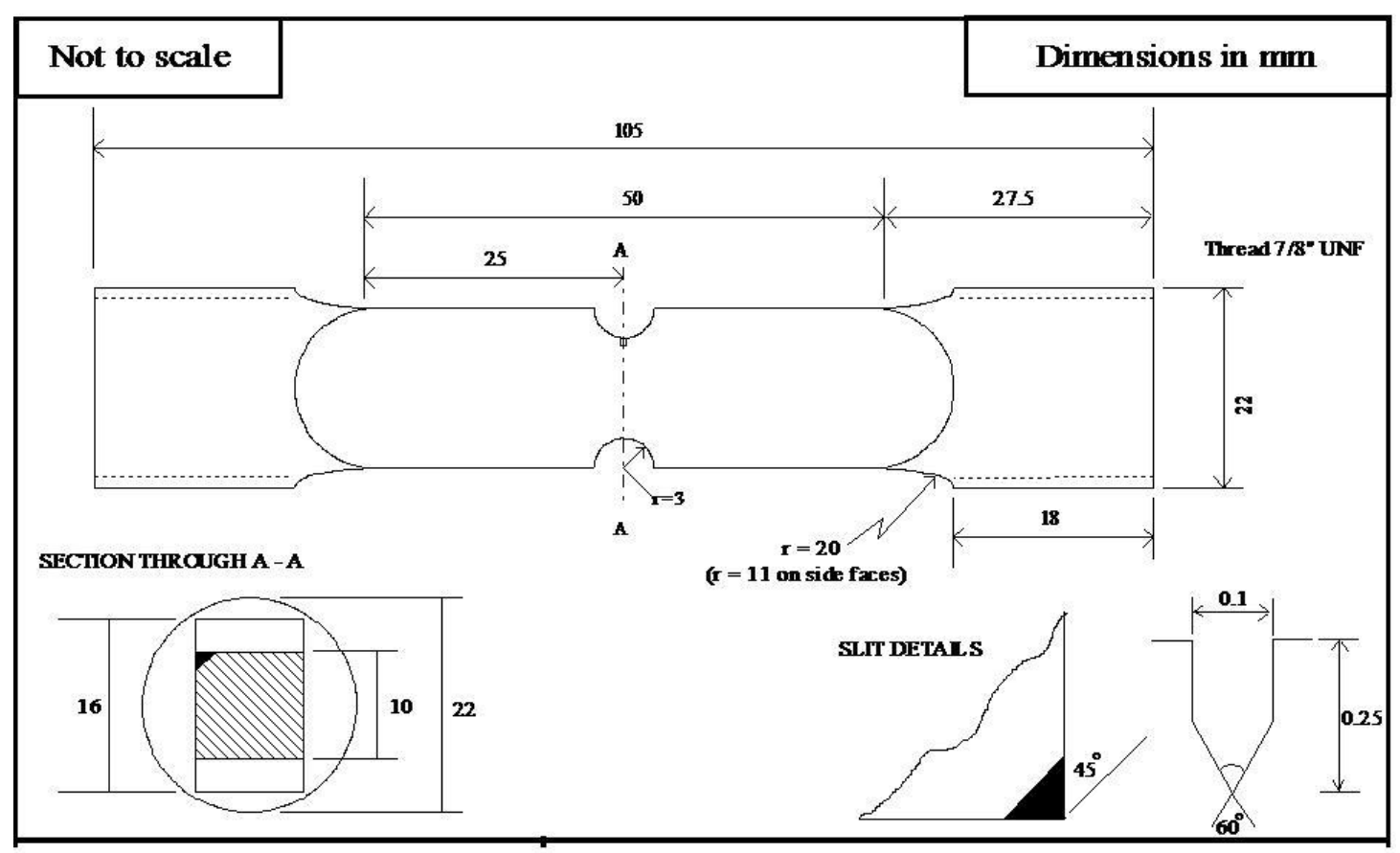

Figure 2: Geometry of Double-edged notch (DEN) specimen, $\mathrm{K}_{\mathrm{t}}=1.9$. 


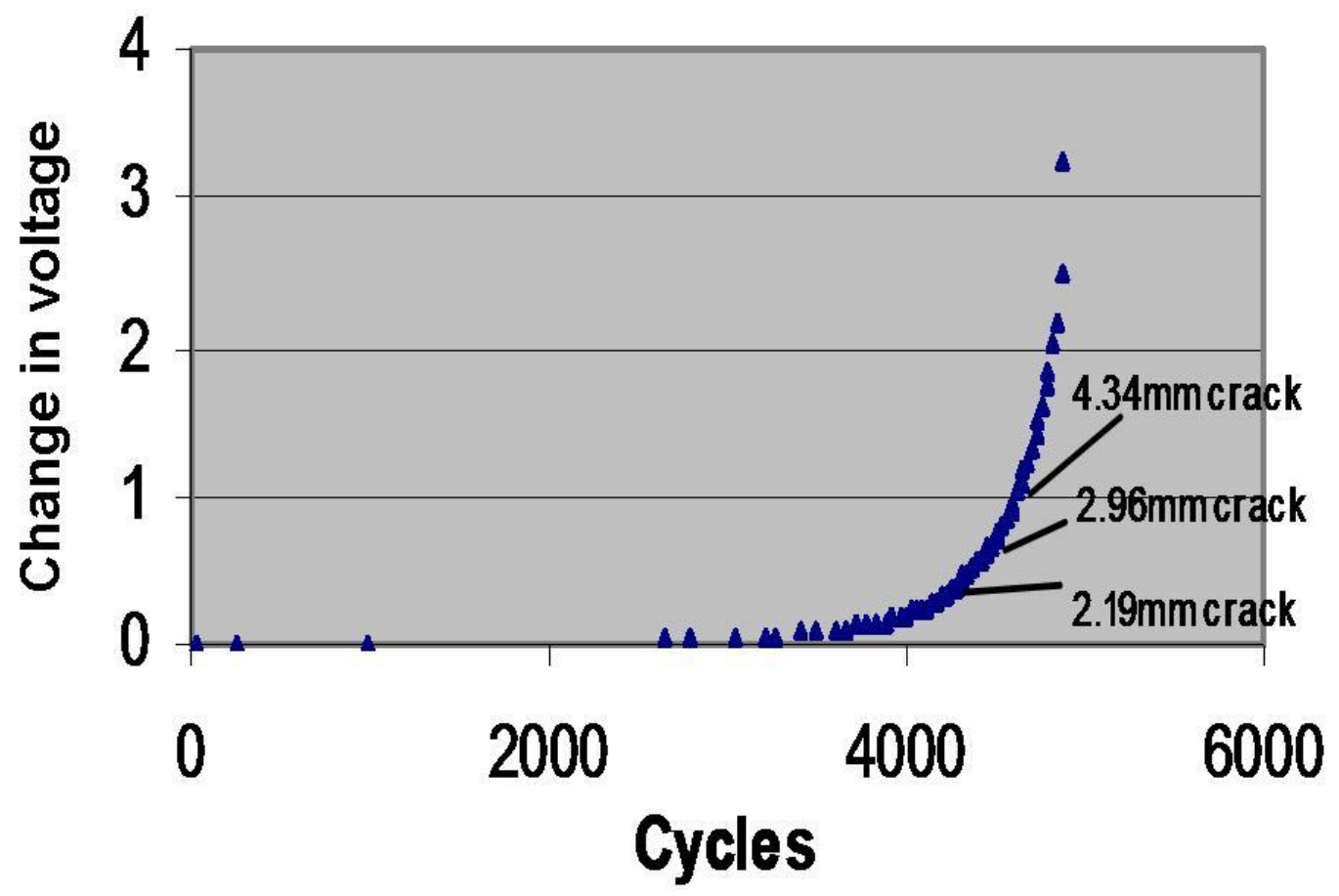

Figure 3: Representation of potential drop data for fatigue testing of a DEN specimen, indicating crack initiation to be approximately $50 \%$ of total life. Indicated crack sizes recorded from previous tested specimens with heat tinting applied. 


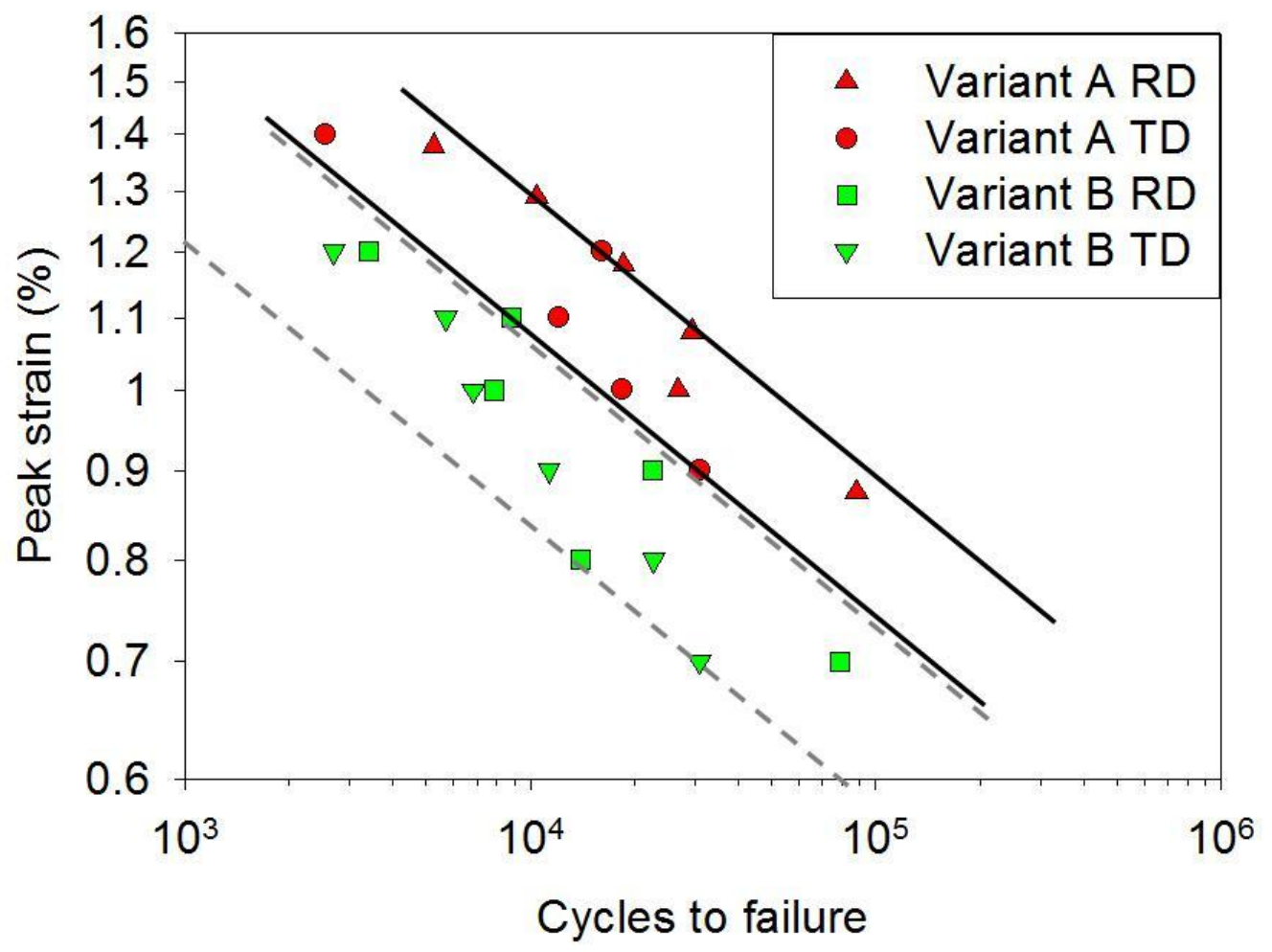

Figure 4: Plots for variants A \& B indicating a variation in strain-life due to texture. 


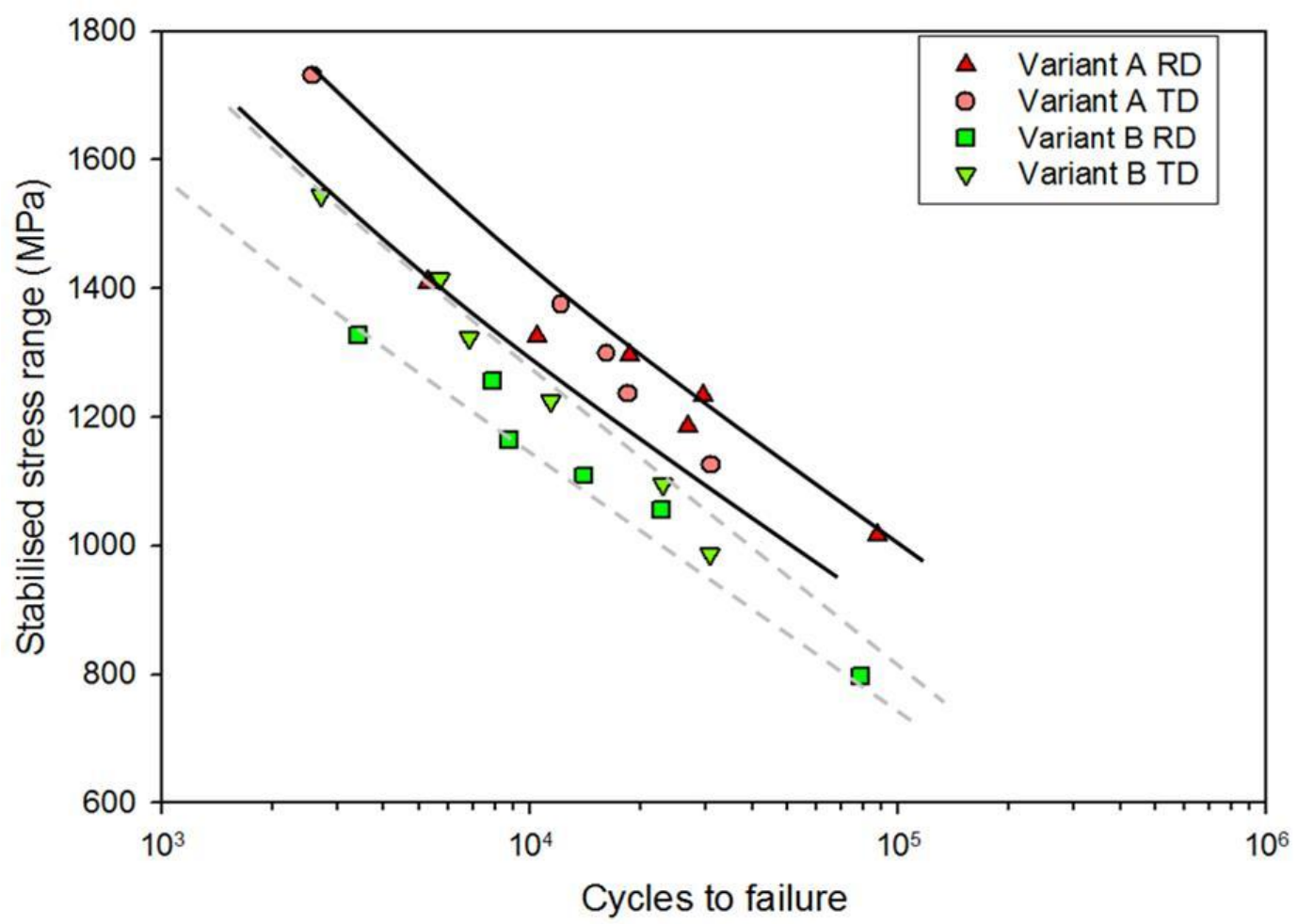

Figure 5: Stabilised stress range vs life in strain control tests, indicating that texture differences are only seen on a strain-life basis. 


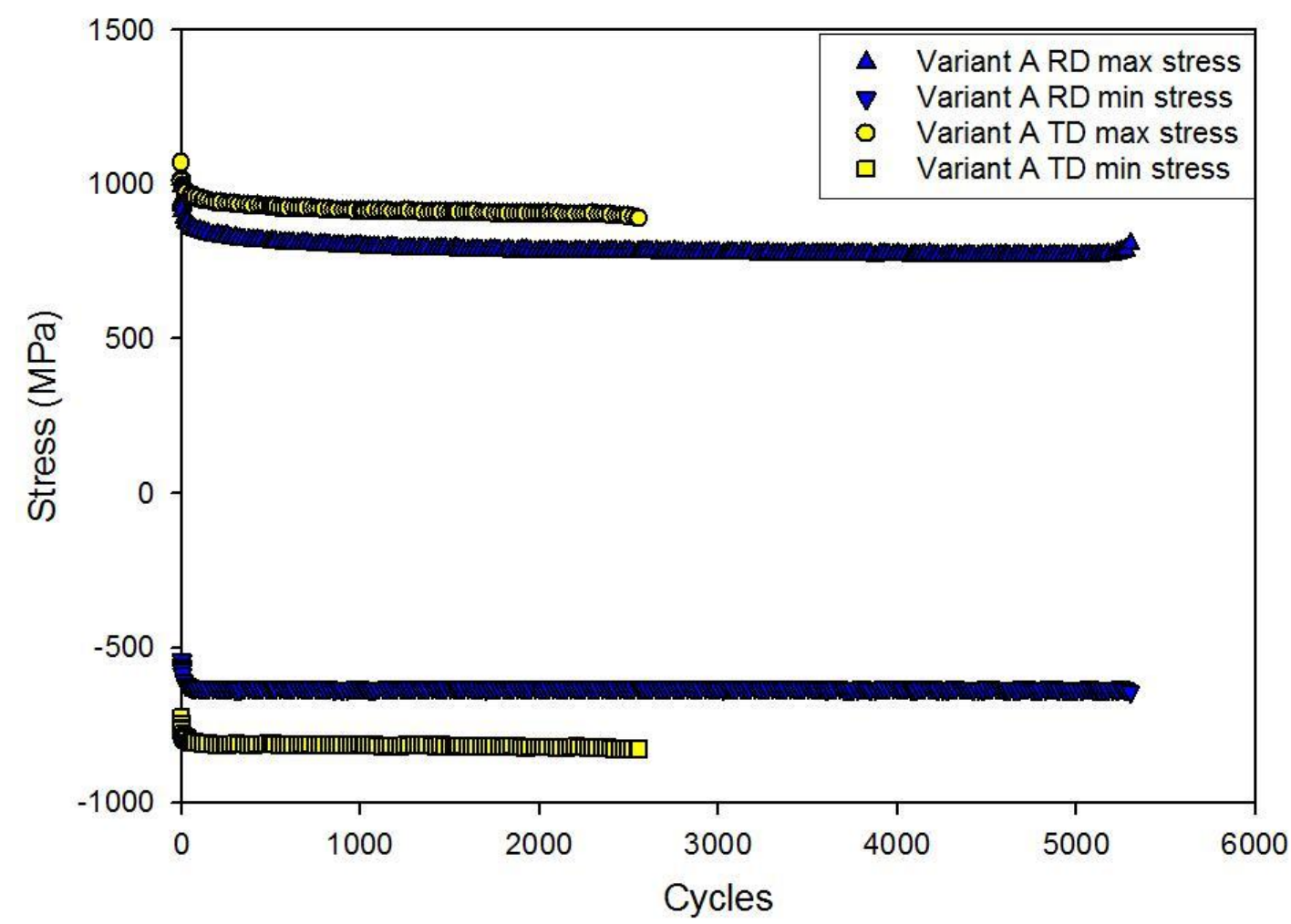

Figure 6: Easier prismatic slip in RD specimens, along with a lower modulus compared with the TD specimens, resulting a lower stabilised stress range in $\mathrm{RD}$ specimens. 


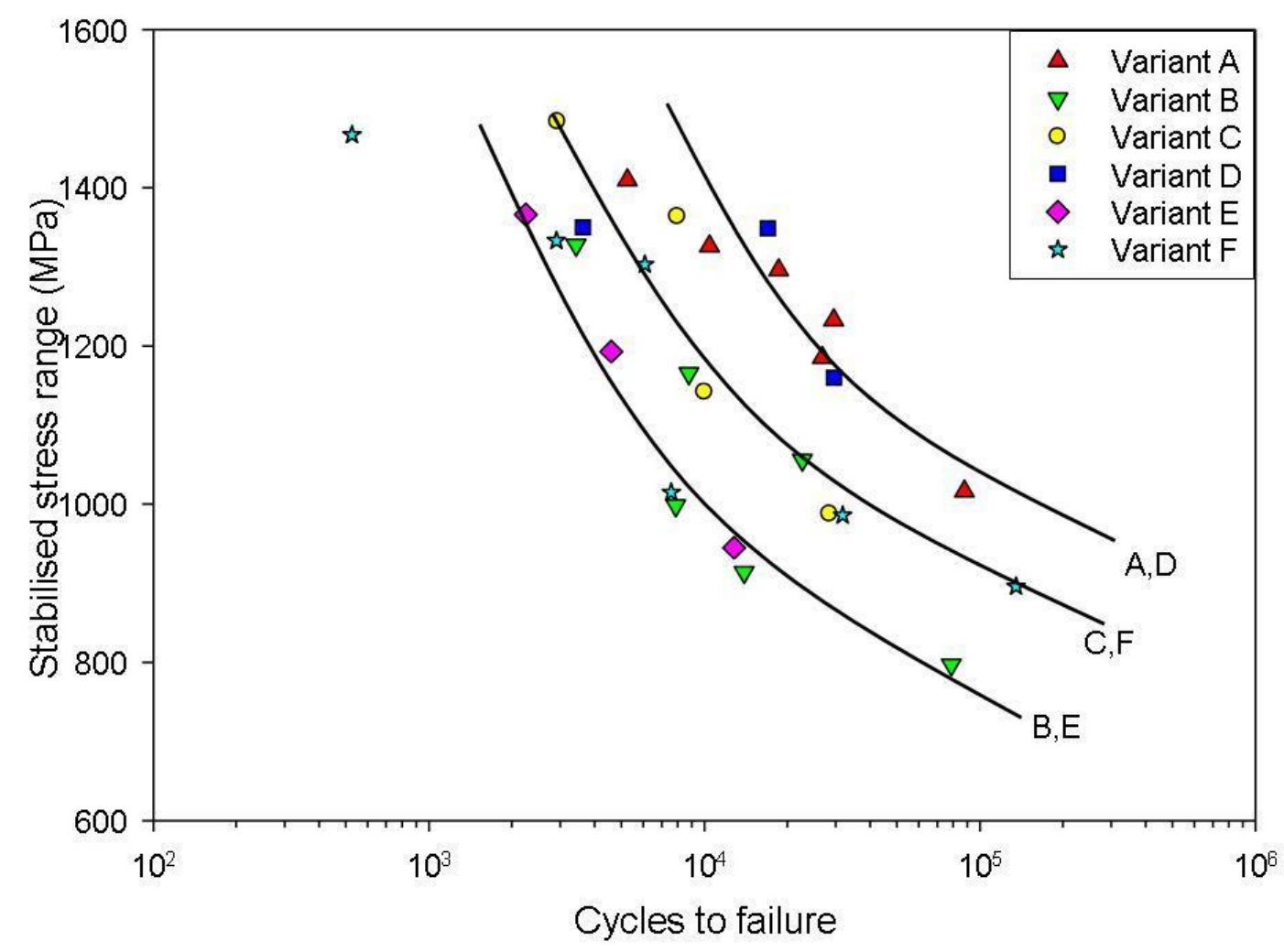

Figure 7: Comparison of fatigue life of various microstructures, indicating that microstructure strongly affects stabilised stress-life. 


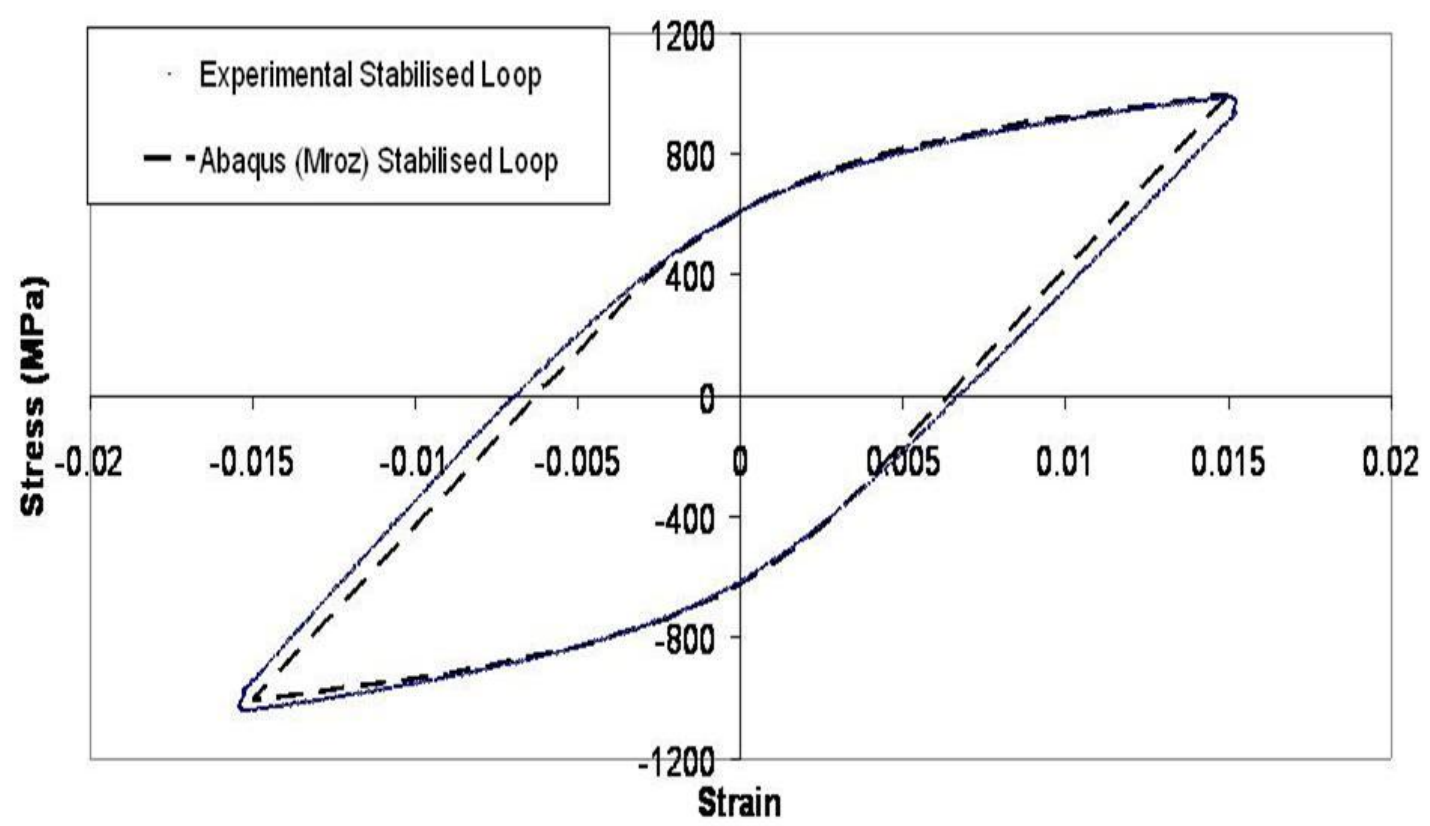

Figure 8: Comparison of ABAQUS stabilised loop predictions with experimental data. 


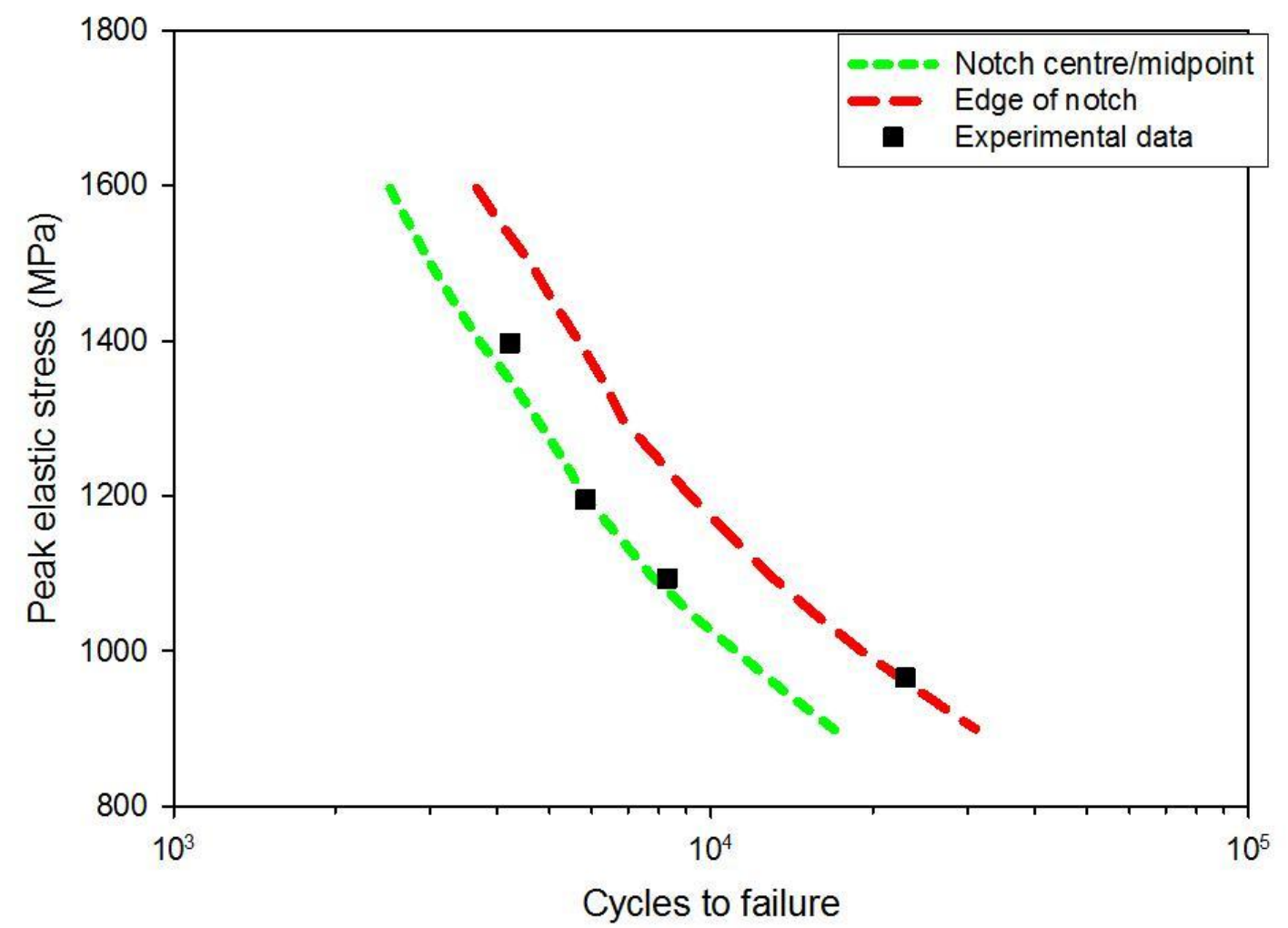

Figure 9: Predictions made by ABAQUS model for initiation life of DEN specimens in variant $\mathrm{C}$ (centre and edge nodes), plotted against experimental data, for which a significant propagation life will occur. 


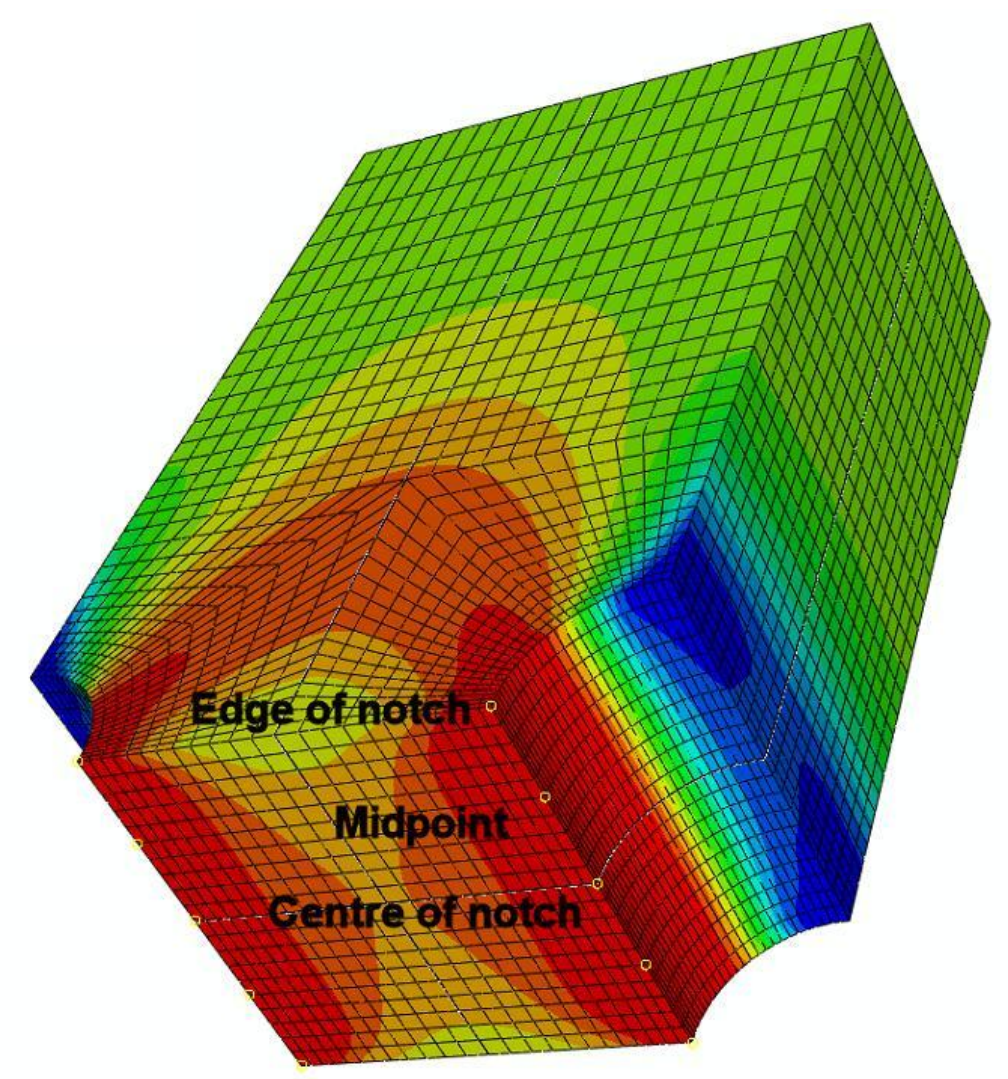

Figure 10: ABAQUS model of DEN specimen, indicating stress concentration at the notch root. Nodes used for predictions in Figure 9 are also indicated 


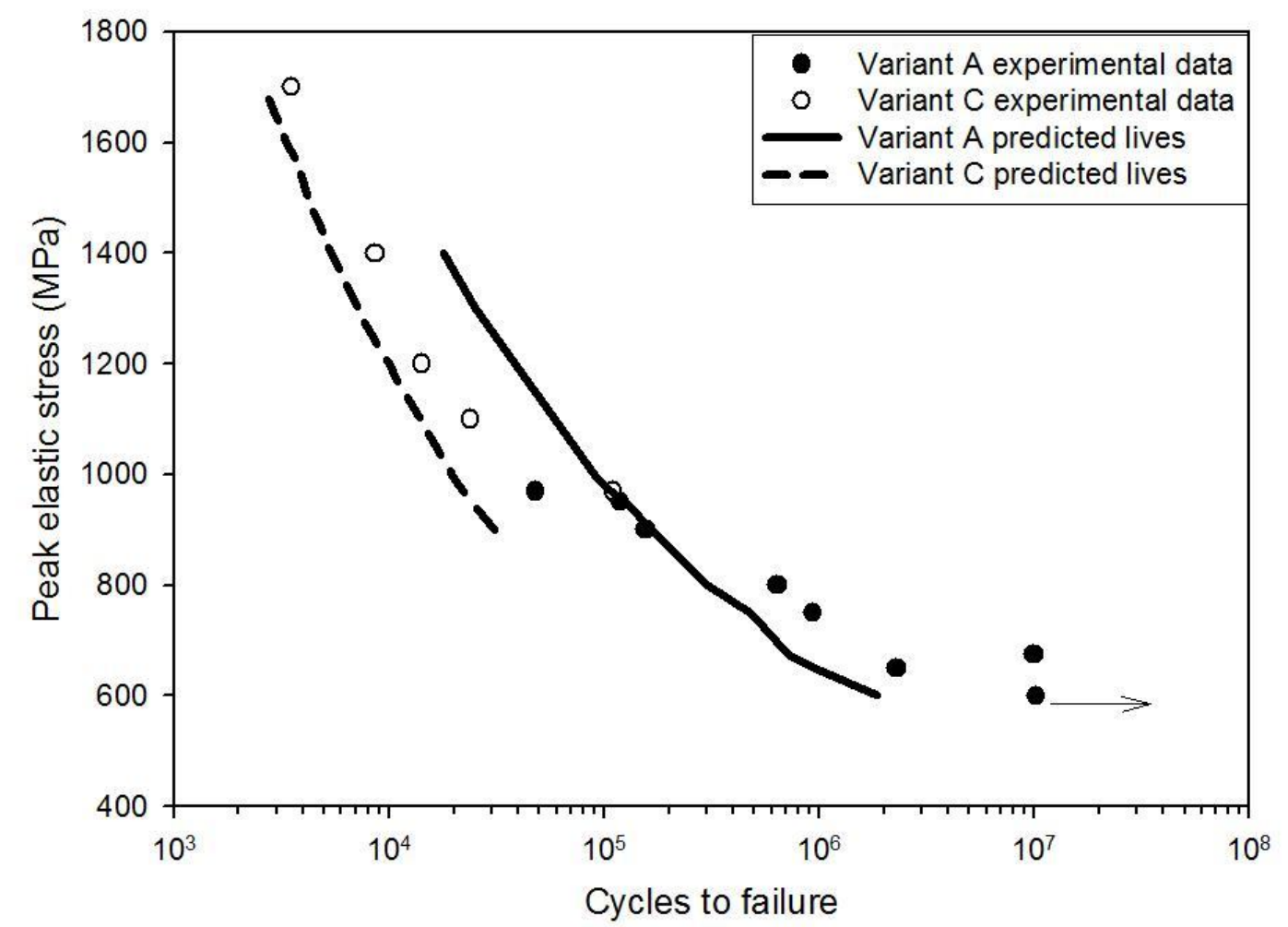

Figure 11: Predicted initiation lives of variants A\&C using Walker method. Experimental data includes a crack propagation phase prior to final failure 


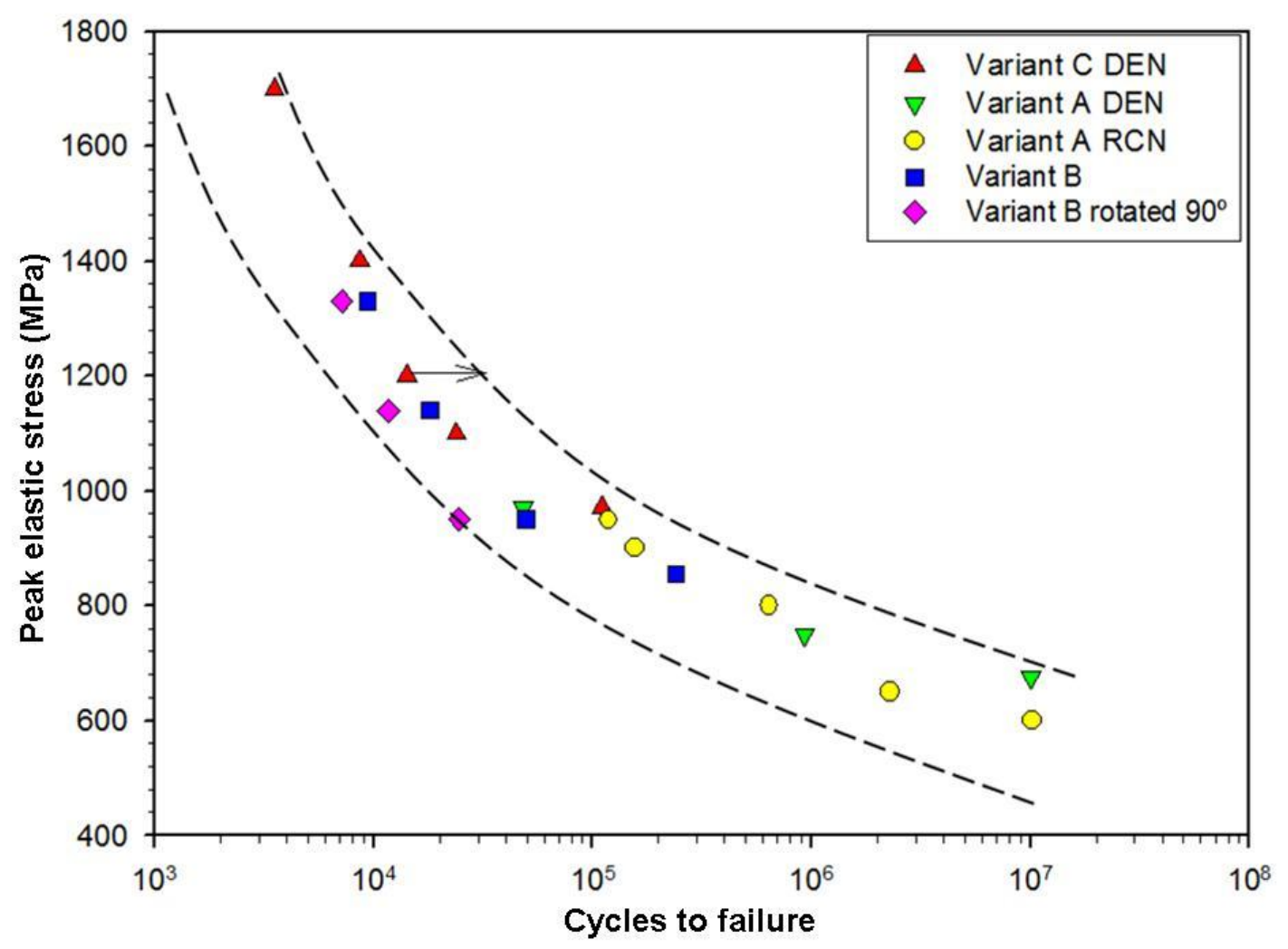

Figure 12: Fatigue life of notched specimens in microstructural variants $A, B \& C$ showing minimal variation 


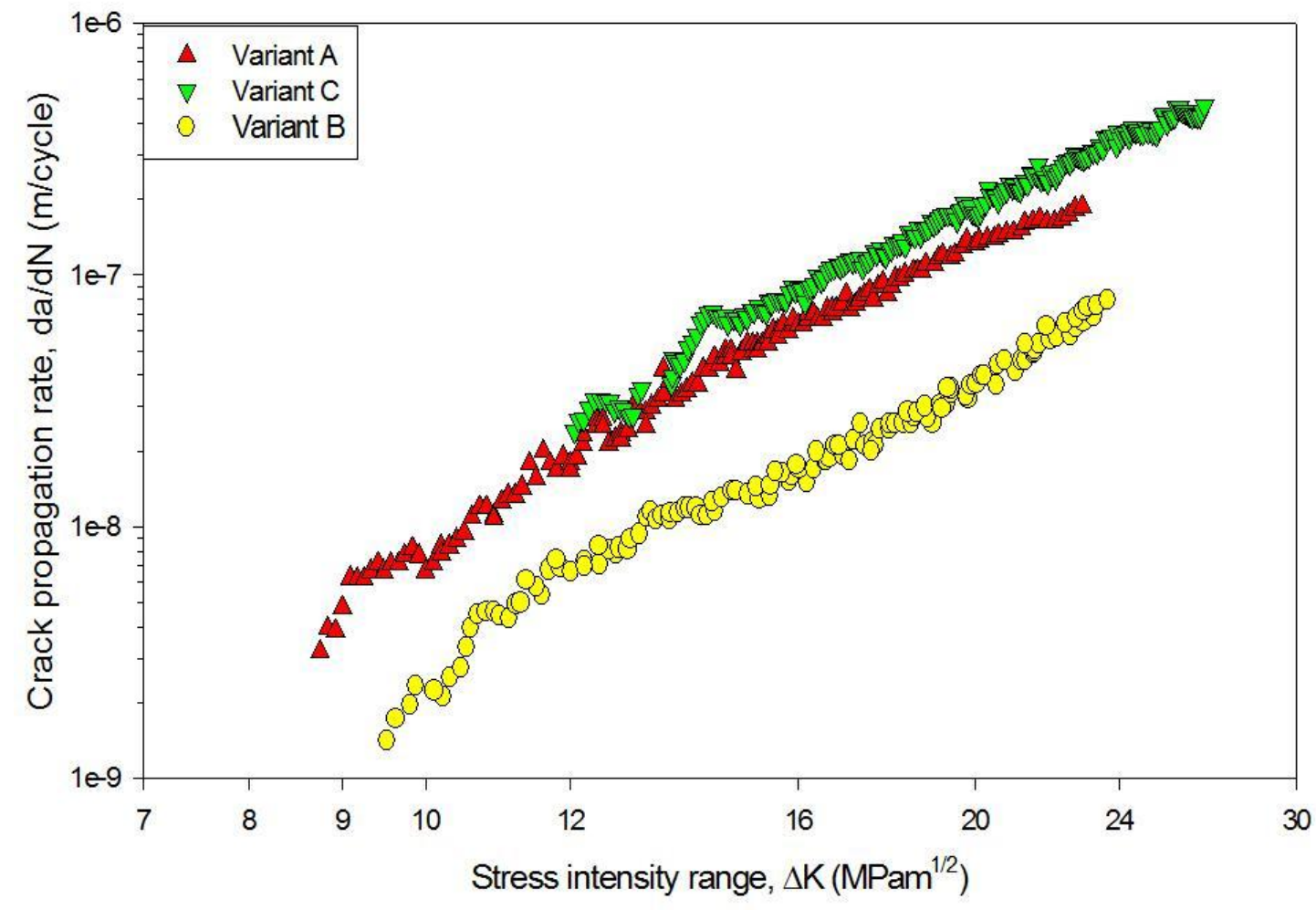

Figure 13: Crack propagation rates of variants $A, B \& C$ indicating significantly faster rate in variant $\mathrm{B}$ 